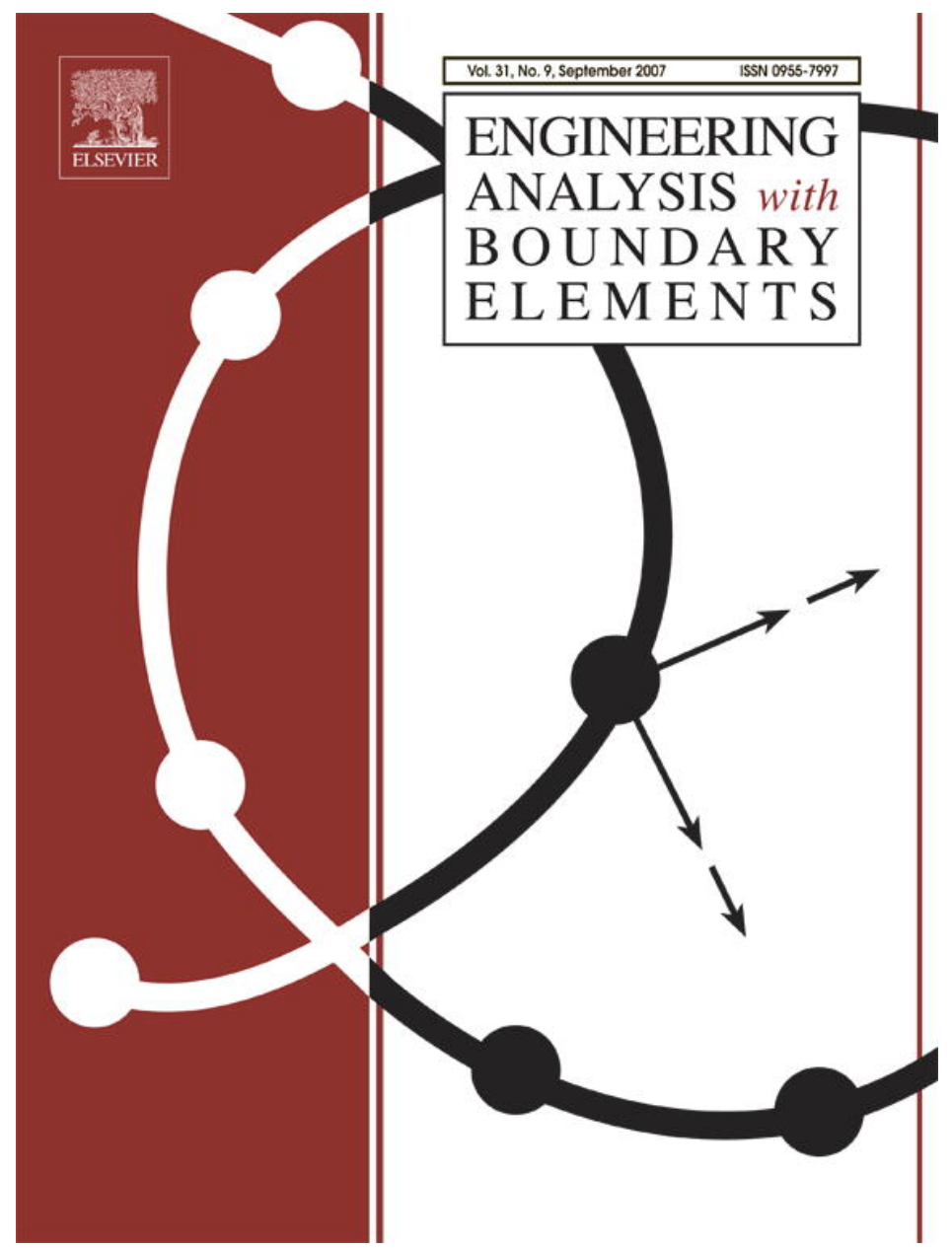

This article was published in an Elsevier journal. The attached copy

is furnished to the author for non-commercial research and education use, including for instruction at the author's institution, sharing with colleagues and providing to institution administration.

Other uses, including reproduction and distribution, or selling or licensing copies, or posting to personal, institutional or third party websites are prohibited.

In most cases authors are permitted to post their version of the article (e.g. in Word or Tex form) to their personal website or institutional repository. Authors requiring further information regarding Elsevier's archiving and manuscript policies are encouraged to visit: 


\title{
Solving potential problems by a boundary-type meshless method-the boundary point method based on BIE
}

\author{
Hang Ma ${ }^{\mathrm{a}}$, Qing-Hua Qin ${ }^{\mathrm{b}, *}$ \\ ${ }^{a}$ Department of Mechanics, College of Sciences, Shanghai University, Shanghai 200444, PR China \\ ${ }^{\mathrm{b}}$ Department of Engineering, Australian National University, ACT 0200, Australia \\ Received 15 September 2006; accepted 1 March 2007 \\ Available online 18 April 2007
}

\begin{abstract}
In this paper, a novel boundary-type meshless method, the boundary point method (BPM), is developed via an approximation procedure based on the idea of Young et al. [Novel meshless method for solving the potential problems with arbitrary domain. $\mathbf{J}$ Comput Phys 2005;209:290-321] and the boundary integral equations (BIE) for solving two- and three-dimensional potential problems. In the BPM, the boundary of the solution domain is discretized by unequally spaced boundary nodes, with each node having a territory (the point is usually located at the centre of the territory) where the field variables are defined. The BPM has both the merits of the boundary element method (BEM) and the method of fundamental solution (MFS), both of these methods use fundamental solutions which are the two-point functions determined by the source and the observation points only. In addition to the singular properties, the fundamental solutions have the feature that the greater the distance between the two points, the smaller the values of the fundamental solutions will be. In particular, the greater the distances, the smaller the variations of the fundamental solutions. By making use of this feature, most of the off-diagonal coefficients of the system matrix will be computed by one-point scheme in the BPM, which is similar to the one in the MFS. In the BPM, the 'moving elements' are introduced by organizing the relevant adjacent nodes tentatively, so that the source points are placed on the real boundary of the solution domain where the resulting weak singular, singular and hypersingular kernel functions of the diagonal coefficients of the system matrix can be evaluated readily by well-developed techniques that are available in the BEM. Thus difficulties encountered in the MFS are removed because of the coincidence of the two points. When the observation point is close to the source point, the integrals of kernel functions can be evaluated by Gauss quadrature over territories.

In this paper, the singular and hypersingular equations in the indirect and direct formulations of the BPM are presented corresponding to the relevant BIE for potential problems, where the indirect formulations can be considered as a special form of the MFS. Numerical examples demonstrate the accuracy of solutions of the proposed BPM for potential problems with mixed boundary conditions where good agreements with exact solutions are observed.
\end{abstract}

(C) 2007 Elsevier Ltd. All rights reserved.

Keywords: Meshless method; Fundamental solution; Boundary integral equation; Singular and hypersingular kernels; Moving element; Boundary point method

\section{Introduction}

Classical numerical methods used to find the solutions of a wide variety of engineering problems make use of some form of discretization. Depending on the scheme of discretization adopted, numerical solution techniques for boundary-value problems can be classified into domaintype methods and boundary-type methods [1]. In recent

\footnotetext{
*Corresponding author. Tel:: +6126125 8274; fax: +61261250506.

E-mail address: qinghua.qin@anu.edu.au (Q.-H. Qin).
}

years, meshless methods, particularly the boundary-type meshless methods, have attracted much attention due to their simplicity to use and wide applicability to varied engineering problems. These are considered as promising numerical methods in computational mechanics. Meshless methods do not require meshes to discretize the solution domain, and the approximate solution is constructed completely based on a set of scattered nodes. Since neither domain nor surface meshing is required, meshless methods have become very popular for engineers in model creation and are important in scientific computation. Several 
domain-type meshless methods, such as the element free Galerkin (EFG) method [2], the reproducing kernel particle method [3], the point interpolation method (PIM) [4], the point interpolation method using radial basis functions [5], the meshless local Petrov-Galerkin (MLPG) method [6], and the smooth particle hydrodynamics (SPH) method [7] have been proposed recently and have achieved remarkable progress in solving a wide range of static and dynamic problems for solids and structures.

For a large class of problems, boundary-type solution methodologies are now well established as viable alternatives to the prevailing domain-type methods because of the computational advantages they offer. In particular, boundary-type methods have the merit of predicting accurate and complete solutions while reducing the dimensionality of any given problem by one and thus simplifying the effort involved in data preparation and computer time. The BEM is the most popular boundarytype solution procedure, formulated in terms of BIE [1]. There are also several boundary-type meshless methods reported where the dimensionality advantage can be retained, corresponding to the BEM. The boundary node method (BNM) represents a coupling between the BIE and the moving least-square approximations $[8,9]$. The Trefftz method is a noteworthy boundary-type meshless method that features conciseness and an ease of performance. The crucial construct of the Trefftz method is the use of a set of trial functions, singular or non-singular, which a priori satisfy the governing differential equation under consideration [10,11]. In the boundary knot method (BKM), a set of non-singular general solutions is employed as trial functions to solve Helmholtz and convection-diffusion equations [12,13]. The merits of using non-singular trial functions such as T-complete functions [14] or general solutions lie in the fact that the collocation and observation points can be coincident and placed on the real boundary of the problem. However, the matrix of the influence coefficients thus formed may be illconditioned or the condition number of the matrix could become large [14] so that the scale of solvable problems would be limited. In the method of fundamental solutions (MFS), singular functions are taken as the trial functions [15-19]. As the singular nature of the fundamental solutions, however, the source points must be placed outside the solution domain to avoid the singularity which forms a fictitious non-physical boundary, or a pseudooffset boundary. Determination of the offset distance from the real physical boundary may become troublesome and ambiguous, especially for engineering problems that involves complicated geometry. If the offset distance is too small, the diagonal coefficients of the influence matrices will diverge because of the singular nature of the fundamental solutions. On the other hand, if the offset boundary is far from the real boundary, the influence matrices may also become ill-conditioned since the condition number of the influence matrix becomes very large. The location of the source and observation points is vital to the accuracy of the solution when implementing the MFS.

A distinct feature in the Trefftz method including the BKM and the MFS is that each of the coefficients of system matrices are computed on only one point (integration-free), compared with element-type methods in which the integration must be performed over elements. This feature, which is usually known as one-point scheme, greatly reduces the computational cost. Stimulated by the work in [10-19], a novel boundary-type meshless methodthe boundary point method (BPM) is here developed via an approximation procedure based on the idea in [20] and the BIE to eliminate the shortcoming of the MFS described above. In the BPM, the boundary of the solution domain is discretized by unequally spaced boundary nodes, with each node having a territory where the field variables are defined. The BPM has both the merits of the BEM and the MFS, as both of these methods use fundamental solutions, the two-point functions. As mentioned before, in addition to the singular properties, the fundamental solutions have the feature that the greater the distance between the two points, the smaller the values of the fundamental solutions. By making use of the feature, most of the off-diagonal coefficients of the system matrix are computed by one-point scheme in the BPM, just like that in the MFS. In order to deal with the singularity, the 'moving elements' are introduced by organizing the relevant adjacent nodes tentatively, so that the source points are placed on the real boundary of the solution domain where the resulting weak singular, singular and hypersingular kernel functions of the diagonal coefficients of the system matrix can be evaluated readily by well-developed techniques used in the BEM. Thus difficulties encountered in the MFS are removed because of the coincidence of the two points. When the observation point is close to the source point, the integrals of kernel functions are evaluated by Gauss quadrature over the specified domains. The introduction of the moving element makes it possible to switch readily between the one-point scheme and Gauss quadrature for evaluating the coefficients of the system matrix, while most of them are evaluated by one-point scheme in the BPM. It is obvious that the derivation procedure in the BPM can be extended to numerous applications while keeping all the merits of the BEM.

\section{Formulations}

\subsection{Basic equations}

The governing equation and the boundary conditions of the boundary-value problem governed by the Laplace equation are given as follows:

$\nabla^{2} u(x)=0, \quad x \in \Omega$,

$u(x)=\bar{u}, \quad x \in \Gamma_{1}$, 
$q(x)=\frac{\partial u}{\partial n}=\bar{q}, \quad x \in \Gamma_{2}$,

where $u$ and $q$ are the potential and its normal derivative (flux), respectively. $\Omega, \Gamma_{1}$ and $\Gamma_{2}$ are the solution domain, the Dilichlet and the Neumann boundaries $\left(\Gamma=\Gamma_{1} \cup \Gamma_{2}\right)$, respectively on which the potential and flux are specified. (-) represents the specified value, $x$ the field point and $n$ the outward unit vector on the boundary. The real physical problems for the Laplace equation include steady temperature fields, potential flow problems, torsion bar problems and Stokes equations of the vorticity transport equations. There are indirect and direct formulations of the BIE [1] that describe the Laplace equation equivalently. The single and the double layer potentials in the indirect formulation for the interior problems are as follows:

$u(y)=\int_{\Gamma} \psi(x) u^{*}(x, y) \mathrm{d} \Gamma(x)$,

$u(y)=[1-\gamma(y)] \varphi(y)-\int_{\Gamma} \varphi(x) \frac{\partial u^{*}(x, y)}{\partial n(x)} \mathrm{d} \Gamma(x)$.

The physical meaning of the above two equations can be explained as the potentials induced from the distributions of the charge $\psi$ and the moment of couple $\varphi$ on $\Gamma$, respectively. In Eqs. (4) and (5), $y$ is the source point, $u^{*}[=$ $\log (1 / r) /(2 \pi)$ in a two-dimensional (2D) problem or $=$ $1 /(4 \pi r)$ in a three-dimensional (3D) problem] is the fundamental solution of Laplace equation, where $r$ is the distance between $x$ and $y$. In addition, $\gamma$ represents the coefficient relating to the free term of singular kernels. $\gamma(y)=1$ when $y \in \Omega$ but $\gamma(y)=0.5$ when $y$ is located on smooth boundaries. Incorporated with Dilichlet boundary conditions, $\psi$ or $\varphi$ can be solved using Eq. (4) or (5). For Neumann boundary conditions, following the normal derivation procedures and by locating $y$ on $\Gamma$, Eqs. (4) and (5) will lead to

$$
\begin{aligned}
q(y)= & {[1-\gamma(y)] \psi(y) } \\
& +\int_{\Gamma} \psi(x) \frac{\partial u^{*}(x, y)}{\partial n(y)} \mathrm{d} \Gamma(x), \quad y \in \Gamma, \\
q(y)=- & -\int_{\Gamma} \varphi(x) \frac{\partial^{2} u^{*}(x, y)}{\partial n(x) \partial n(y)} \mathrm{d} \Gamma(x), \quad y \in \Gamma .
\end{aligned}
$$

In the present work, Eqs. (4) and (6) are termed as the first equations, and Eqs. (5) and (7) are termed as the second equations, respectively, of the indirect formulations. When $y \in \Gamma$ and $y$ and $x$ are coincident, the kernels become weak singular in Eq. (4), singular in Eqs. (5) and (6) and hypersingular in Eq. (7), respectively, which require special attention to numerical implementation.

The direct formulation of BIE has recently gained popularity among engineers and scientists, since the problem unknowns are the same as the real physical variables that govern the equations. With the terminology in the present work, the first (or conventional, singular) and the second (hypersingular) integral equations of direct formulations are expressed as follows [1]:

$$
\begin{gathered}
\gamma(y) u(y)+\int_{\Gamma} u(x) \frac{\partial u^{*}(x, y)}{\partial n(x)} \mathrm{d} \Gamma(x) \\
=\int_{\Gamma} q(x) u^{*}(x, y) \mathrm{d} \Gamma(x), \\
\gamma(y) q(y)+\int_{\Gamma} u(x) \frac{\partial^{2} u^{*}(x, y)}{\partial n(x) \partial n(y)} \mathrm{d} \Gamma(x) \\
=\int_{\Gamma} q(x) \frac{u^{*}(x, y)}{\partial n(y)} \mathrm{d} \Gamma(x) .
\end{gathered}
$$

The second or hypersingular BIE (9) is derived from a differentiated version of the first or conventional BIE (8). The hypersingular BIE has diverse important applications such as evaluation of boundary stresses and fracture mechanics. The first and the second BIE in the indirect and direct formulations are the basis of the BEM. With the impetus of the MFS [15-20] as inspiration, a novel boundary-type meshless method, the BPM, can be derived based on the BIE via the approximation procedure described in the following subsection.

\subsection{The boundary point method}

Discretization in the BPM is simple to implement but slightly different from that in the BEM using constant boundary elements. Suppose that $N$ nodes are placed on a smooth section of the boundary $\Gamma$, with each node being the centroid of a territory, $\Delta \Gamma_{m}(m=1,2, \ldots, N)$, which is locally smooth. A territory is a segment of curved line in $2 \mathrm{D}$ or a piece of curved surface in 3D such that the node in the BPM locates on the $\Gamma$. The summation of all the territories form the total boundary, that is,

$\Gamma=\bigcup_{m=1}^{N} \Delta \Gamma_{m}, \quad \Delta \Gamma_{m}= \begin{cases}l_{m} & (2 \mathrm{D}), \\ a_{m} & \text { (3D), }\end{cases}$

where $l_{m}$ and $a_{m}$ denote the curved length and the curved area of the territory of the $m$ th node, respectively. The boundary variables are assumed to be constant over a territory. However, the difference between a territory in the BPM and a constant element in the BEM can be seen clearly in that an element is a segment of straight line in $2 \mathrm{D}$ or a piece of plane surface in 3D so that, in general, the node in the BEM does not locate on the real $\Gamma$. However, the node in the BPM does locate on the real $\Gamma$. In addition, the curved line boundary in 2D and the curved surface boundary can be correctly described by the use of moving elements when necessary, as discussed in the following section.

By dividing $\Gamma$ into territories, the first Eqs. (4) and (6) in the indirect formulation can be written in discrete form as follows:

$\bar{u}\left(y_{i}\right)=\sum_{m=1}^{N} \psi\left(x_{m}\right) \int_{\Delta \Gamma_{m}} u^{*}\left(x, y_{i}\right) \mathrm{d} \Gamma(x), \quad y_{i} \in \Gamma_{1}$, 


$$
\begin{aligned}
\bar{q}\left(y_{i}\right)= & 0.5 \psi\left(y_{i}\right)+\sum_{m=1}^{N} \psi\left(x_{m}\right) \\
& \times \int_{\Delta \Gamma_{m}} \frac{\partial u^{*}\left(x, y_{i}\right)}{\partial n\left(y_{i}\right)} \mathrm{d} \Gamma(x), \quad y_{i} \in \Gamma_{2}
\end{aligned}
$$

and the second Eqs. (5) and (7) in the indirect formulation can be written in discrete form as follows:

$$
\begin{aligned}
\bar{u}\left(y_{i}\right)= & 0.5 \varphi\left(y_{i}\right)-\sum_{m=1}^{N} \varphi\left(x_{j}\right) \\
& \times \int_{\Delta \Gamma_{m}} \frac{\partial u^{*}\left(x, y_{i}\right)}{\partial n(x)} \mathrm{d} \Gamma(x), \quad y_{i} \in \Gamma_{1}, \\
\bar{q}\left(y_{i}\right)= & \sum_{m=1}^{N} \varphi\left(x_{j}\right) \int_{\Delta \Gamma_{m}} \frac{\partial^{2} u^{*}\left(x, y_{i}\right)}{\partial n(x) \partial n\left(y_{i}\right)} \mathrm{d} \Gamma(x), \quad y_{i} \in \Gamma_{2} .
\end{aligned}
$$

It is known that the form of Eqs. (11)-(14) are also constructed from the first and the second equations of the indirect BEM formulations using constant elements so the integrals of kernel functions over element $m$ are defined as follows:

$$
\begin{aligned}
G_{i m} & =\int_{\Delta \Gamma_{m}} u^{*}\left(x, y_{i}\right) \mathrm{d} \Gamma(x), \\
F_{i m} & =\int_{\Delta \Gamma_{m}} \frac{\partial u^{*}\left(x, y_{i}\right)}{\partial n\left(y_{i}\right)} \mathrm{d} \Gamma(x), \\
T_{i m} & =\int_{\Delta \Gamma_{m}} \frac{\partial u^{*}\left(x, y_{i}\right)}{\partial n(x)} \mathrm{d} \Gamma(x), \\
H_{i m} & =\int_{\Delta \Gamma_{m}} \frac{\partial^{2} u^{*}\left(x, y_{i}\right)}{\partial n(x) \partial n\left(y_{i}\right)} \mathrm{d} \Gamma(x) .
\end{aligned}
$$

Eqs. (11)-(14) can be further written in compact form as

$$
\begin{aligned}
& \bar{u}\left(y_{i}\right)=\sum_{m=1}^{N} G_{i m} \psi_{m}, \\
& \bar{q}\left(y_{i}\right)=\sum_{m=1}^{N}\left(F_{i m}+0.5 \delta_{i m}\right) \psi_{m},
\end{aligned}
$$

$\bar{u}\left(y_{i}\right)=-\sum_{m=1}^{N}\left(T_{i m}-0.5 \delta_{i m}\right) \varphi_{m}$,

$\bar{q}\left(y_{i}\right)=\sum_{m=1}^{N} H_{i m} \varphi_{m}$,

where $\psi_{m}=\psi\left(x_{m}\right)$ and $\varphi_{m}=\varphi\left(x_{m}\right)$ are the boundary unknowns to be solved. When $x$ and $y$ are coincident $(i=m)$, the integrals are weakly singular in Eq. (15a), strongly singular in Eqs. (15b), (15c) and hypersingular in Eq. (15d), which constitute the principal diagonal terms of the system matrix. The strongly and hypersingular boundary integrals can be evaluated in the sense of Cauchy principal values and Hadamard finite part values, respectively. This issue is discussed as follows.
When $i \neq m$ or $y \in \Omega$, the kernel functions in the integrals (15) are regular and therefore are easy to evaluate if the distances $r$ are relatively not too small. In this case, Brebbia suggested that 4-point Gauss quadrature would have sufficient accuracy for constant elements in the BEM [1]. However, as the kernel functions in integrals (15), in this case, constitute most of the off-diagonal terms of the system matrix, computational cost can be significantly reduced by the use of one-point scheme. In addition, if a kernel has a singular order of $O\left(r^{-s}\right)$, where $s$ is an integer, the decaying order of the integral values of this kernel function is also $s$ with the increase of $r$. Consequently, the variation of the kernel will have the order of $O\left(r^{-s-1}\right)$ if $r$ is relatively not too small. Based on these properties, the reasonable accuracy in evaluating the integrals (15) can be achieved using one-point scheme only. In other words, the value of kernel function can be well represented by the value at the node which is located at the centre of the corresponding territory in the BPM, since the variation of the kernel along the territory becomes negligibly small in this case. Therefore, if the distance $r$ is relatively not too small, the one-point scheme will provide reasonably accurate integrals of kernel functions using the following approximations:

$G_{i m} \approx u^{*}\left(x_{m}, y_{i}\right) \Delta \Gamma_{m}$,

$F_{i m} \approx \frac{\partial u^{*}\left(x_{m}, y_{i}\right)}{\partial n\left(y_{i}\right)} \Delta \Gamma_{m}$

$T_{i m} \approx \frac{\partial u^{*}\left(x_{m}, y_{i}\right)}{\partial n(x)} \Delta \Gamma_{m}$

$H_{i m} \approx \frac{\partial^{2} u^{*}\left(x_{m}, y_{i}\right)}{\partial n(x) \partial n\left(y_{i}\right)} \Delta \Gamma_{m}$,

where $\Delta \Gamma_{m}$ is defined by Eq. (10). It can be seen that the formulations (16)-(19) as well as the one-point scheme in the BPM are very similar to that of the MFS. The use of boundary elements is unnecessary. The feasibility of using one-point scheme has been demonstrated by the successful performance of a variant of the MFS [20].

The first and the second equations for the direct formulations in the BPM can be, respectively, derived from Eqs. (8) and (9) by using the same approximation procedures as

$\sum_{m=1}^{N}\left(T_{i m}+0.5 \delta_{i m}\right) u_{m}=\sum_{m=1}^{N} G_{i m} q_{m}$

$\sum_{m=1}^{N} H_{i m} u_{m}=\sum_{m=1}^{N}\left(F_{i m}-0.5 \delta_{i m}\right) q_{m}$.

Incorporating the boundary conditions (2) and (3), and rearranging the boundary known and unknown variables is usually done in the BEM [1], either Eq. (21) or Eq. (22) can be written in matrix form as

$\mathbf{A x}=\mathbf{b}$, 
where the system matrix $\mathbf{A}$ is not symmetric. This is also true for most boundary-type methods based on BIE such as BEM and BNM. However, the approach presented in [21] for obtaining a symmetric $\mathbf{A}$ gives a potential for further study to see if the Galerkin formulation could be used to obtain a symmetric system matrix for the present BPM.

\subsection{The accuracy of one-point scheme}

It is crucial for the proposed BPM that the accuracy of one-point scheme for the values of the kernel functions can be verified. As shown in Fig. 1, the kernel functions are to be evaluated over a territory on $\Gamma$ with a length $l$ and outward normal $n(x) . r_{0}$ is the distance from the source point $y$ to the observation point $x$ or the centre of the territory. The kernel functions are evaluated by both onepoint scheme at the point $x$ and eight-point Gaussian quadrature over the territory. Various kernel function values and absolute errors with different $\theta$ (Fig. 1) are compared in Figs. 2 and 3, respectively, as a function of relative distance, $r_{0} / l$.

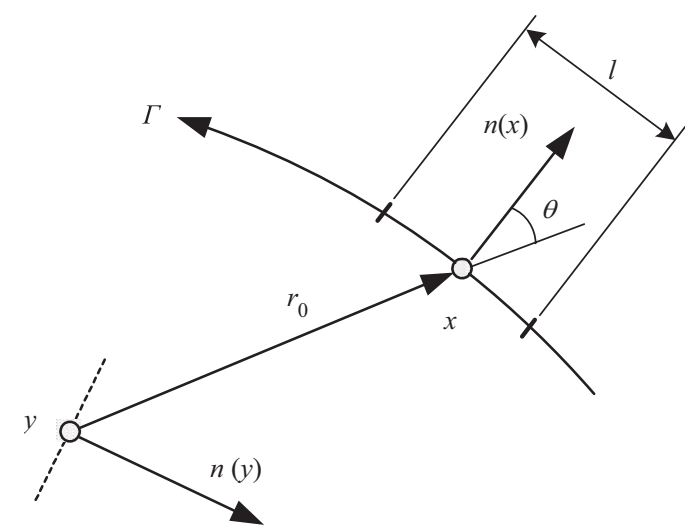

Fig. 1. Schematics for the comparison of one-point scheme for the kernel functions with that of Gaussian quadrature in the 2D.

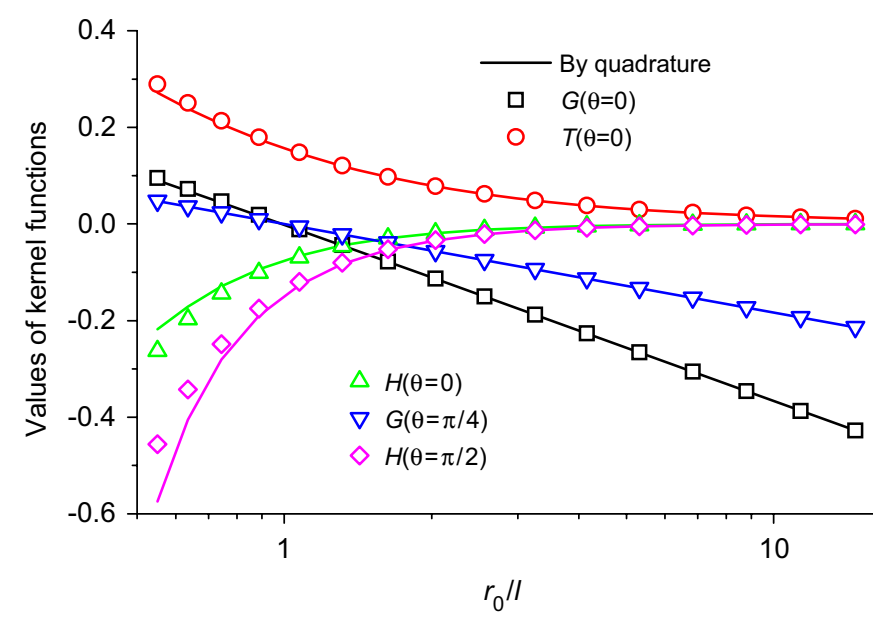

Fig. 2. Comparison of various kernel functions between one-point scheme and Gaussian quadrature as a function of relative distance, $r_{0} / l$.

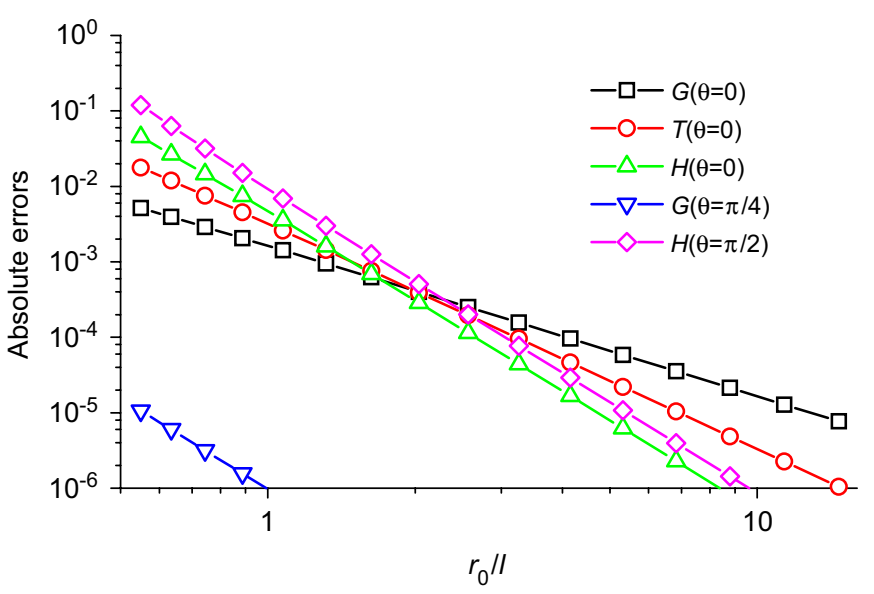

Fig. 3. Comparison of absolute errors between one-point scheme and Gaussian quadrature as a function of relative distance, $r_{0} / l$.

It can be seen that the greater the relative distance is, the smaller the value is, a feature stemming from the properties of fundamental solutions. The corresponding errors of the 2D kernel functions are shown in Fig. 3. It also can be seen that the greater the relative distance is, the smaller the error between the kernel functions by the two algorithms are, a feature also stemming from the properties of fundamental solutions. When the relative distance $r_{0} / l$ exceeds 2 , the difference of the values of fundamental solution from the two algorithms is negligibly small, in which the kernel functions can be computed suitably by one-point scheme. The use of Gauss quadrature is necessary only within the range of $r_{0} / l \leqslant 2$. A similar phenomenon can also be observed in the $3 \mathrm{D}$ case.

\subsection{The moving elements}

As can be seen from Sections 2.2 and 2.3, computational cost can be significantly saved through the use of one-point scheme in evaluating the integrals (15) when the distance $r$ is relatively not too small, which constitute most of the offdiagonal terms of the system matrix in the BPM. However, in some cases the distance between the collocation and field points is relatively small, or they may even be coincident. In this case, the integrals (15) constitute principal and subprincipal diagonal terms of the system matrix. The manner in evaluating these integrals plays an important role in achieving high computational accuracy in the BPM, although their quantity is very small compared with the off-diagonal terms.

Noticing that the adjacent boundary nodes describe the local features of the boundary such as position, curvature and direction, the 'moving elements' can be introduced by organizing the relevant adjacent nodes over which the treatment of singularity and Gauss quadrature can be carried out for evaluating the integrals of kernel functions. As shown in Fig. 4, a 2D domain has been discretized by boundary nodes where the source and observation points are numbered by $i$ and $m$, respectively. In Fig. 1, each node, 


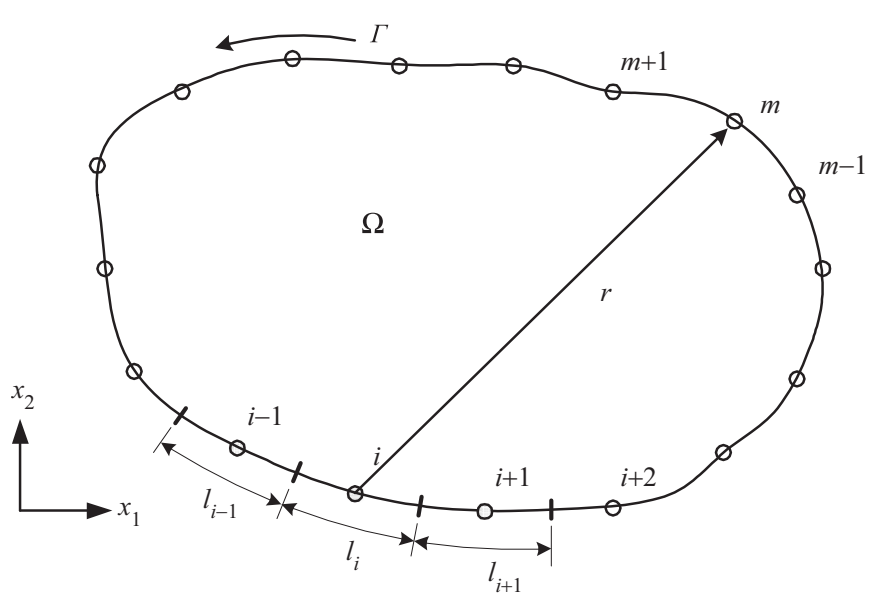

Fig. 4. Schematics of the 'moving element' in the $2 \mathrm{D}$ case.

say node $i$, has a territory $\Delta \Gamma_{i}$ with a length $l_{i}$. In general, a moving element is constructed by three adjacent points. For example, if $m=i$, the moving element is constructed by the nodes $i-1, i$ and $i+1$. In this case, the integral of kernel functions is singular and constitutes the principal diagonal terms of the system matrix. If $m=i+1$, the moving element is then constructed by the nodes $i, i+1$ and $i+2$. In this case, the integrals of kernel functions are regular and constitute the near diagonal terms of the system matrix (see subsequent discussion for more detail). Otherwise, if the difference between the values $i$ and $m$ is not small, the moving element is no longer required. In this case, the integral of kernel functions is regular and constitutes the off-diagonal terms of the system matrix, which can be evaluated by one-point scheme.

The local coordinate system of the three-point moving element for the node of interest, $k$, is shown in Fig. 5a, where $\xi_{j}(j=-1,+1)$ represent the dimensionless distances from the nodes $k-j$ and $k+j$ to the collocation node $k$ in the local coordinate, respectively, as follows:

$\xi_{-1}=1+l_{k-1} / l_{k}$

$\xi_{1}=1+l_{k+1} / l_{k}$,

where $l_{k}$ denotes the territory of each node. With the local coordinate system defined in Fig. 5a and Eq. (24), it is easy to write the shape functions for each node of the threepoint moving element as follows:

$\phi^{k-1}=\frac{1}{\xi_{-1}\left(\xi_{-1}+\xi_{1}\right)} \xi\left(\xi-\xi_{1}\right)$,

$\phi^{k}=\frac{-1}{\xi_{-1} \xi_{1}}\left(\xi+\xi_{-1}\right)\left(\xi-\xi_{1}\right)$,

$\phi^{k+1}=\frac{1}{\xi_{1}\left(\xi_{-1}+\xi_{1}\right)} \xi\left(\xi+\xi_{-1}\right)$.

The integration span for the moving element is $[-1,+1]$ around the collocation node, corresponding to the territory of the node. It can be seen from the discussion above that moving elements are suitable for describing curved a

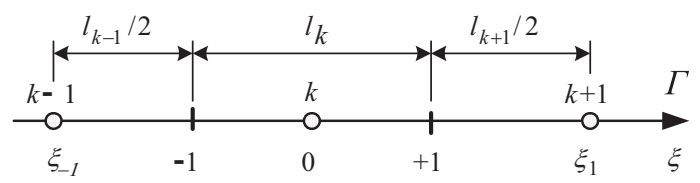

b

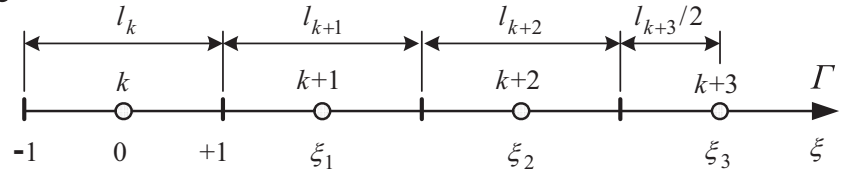

C

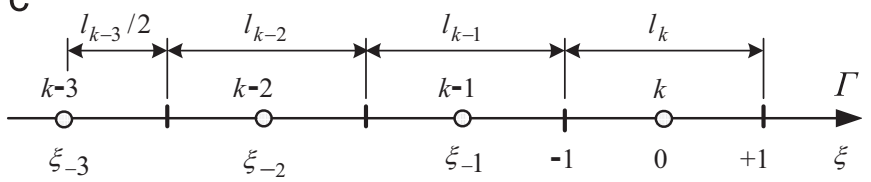

Fig. 5. The local coordinates of a 2D 'moving element' constructed by (a) three-points, (b) and (c) four-points.

boundaries. When the location of the source node closes to a corner of the boundary, four-point moving elements can be constructed. Two local coordinate systems of four-point moving elements are shown in Figs. $5 \mathrm{~b}$ and c, respectively. The purpose of using four adjacent nodes to construct an element is to maintain, approximately, the same accuracy as that of the three-point element, because part of the integration span $[-1,+1]$ for the four-point element around the node of interest is determined by extrapolation (Fig. 5b). In Fig. 5b, $\xi_{j}(j=1,2,3)$ represent the dimensionless distances from the nodes $k+j$ to the collocation node $k$ in the local coordinate system and are defined as follows:

$\xi_{1}=1+l_{k+1} / l_{k}$,

$\xi_{2}=1+\left(2 l_{k+1}+l_{k+2}\right) / l_{k}$,

$\xi_{3}=1+\left(2 l_{k+1}+2 l_{k+2}+l_{k+3}\right) / l_{k}$.

With the local coordinate system defined in Fig. $5 \mathrm{~b}$ as well as Eq. (26), the shape functions for each node of the fourpoint moving element are written as follows:

$\phi^{k}=\frac{-1}{\xi_{1} \xi_{2} \xi_{3}}\left(\xi-\xi_{1}\right)\left(\xi-\xi_{2}\right)\left(\xi-\xi_{3}\right)$,

$\phi^{k+1}=\frac{1}{\xi_{1}\left(\xi_{1}-\xi_{2}\right)\left(\xi_{1}-\xi_{3}\right)} \xi\left(\xi-\xi_{2}\right)\left(\xi-\xi_{3}\right)$,

$\phi^{k+2}=\frac{1}{\xi_{2}\left(\xi_{2}-\xi_{1}\right)\left(\xi_{2}-\xi_{3}\right)} \xi\left(\xi-\xi_{1}\right)\left(\xi-\xi_{3}\right)$,

$\phi^{k+3}=\frac{1}{\xi_{3}\left(\xi_{3}-\xi_{1}\right)\left(\xi_{3}-\xi_{2}\right)} \xi\left(\xi-\xi_{1}\right)\left(\xi-\xi_{2}\right)$.

Similarly, in Fig. $5 \mathrm{c}, \xi_{-j}(j=1,2,3)$ represent the dimensionless distances from the nodes $k-j$ to the collocation node $k$ in the local coordinate and are defined as follows:

$\xi_{-1}=1+l_{k-1} / l_{k}$

$\xi_{-2}=1+\left(2 l_{k-1}+l_{k-2}\right) / l_{k}$, 
$\xi_{-3}=1+\left(2 l_{k-1}+2 l_{k-2}+l_{k-3}\right) / l_{k}$.

With the local coordinate system defined in Fig. $2 \mathrm{c}$ as well as Eq. (28), the shape functions for each node of the fourpoint moving element are written as follows:

$\phi^{k-3}=\frac{-1}{\xi_{-3}\left(\xi_{-3}-\xi_{-1}\right)\left(\xi_{-3}-\xi_{-2}\right)} \xi\left(\xi+\xi_{-1}\right)\left(\xi+\xi_{-2}\right)$,

$\phi^{k-2}=\frac{-1}{\xi_{-2}\left(\xi_{-2}-\xi_{-1}\right)\left(\xi_{-2}-\xi_{-3}\right)} \xi\left(\xi+\xi_{-1}\right)\left(\xi+\xi_{-3}\right)$,

$\phi^{k-1}=\frac{-1}{\xi_{-1}\left(\xi_{-1}-\xi_{-2}\right)\left(\xi_{-1}-\xi_{-3}\right)} \xi\left(\xi+\xi_{-2}\right)\left(\xi+\xi_{-3}\right)$,

$\phi^{k}=\frac{1}{\xi_{-1} \xi_{-2} \xi_{-3}}\left(\xi+\xi_{-1}\right)\left(\xi+\xi_{-2}\right)\left(\xi+\xi_{-3}\right)$.

For $3 \mathrm{D}$ cases, the surface moving elements can be constructed in a similar way along the two directions over the boundary surface. The local coordinate system for a nine-point surface moving element is shown in Fig. 6. The shape function for each node of the surface element is defined by the product of the corresponding 2D shape functions in Eq. (25) as follows:

$$
\begin{aligned}
& \psi^{s, t}(\xi, \eta)=\phi^{s}(\xi) \phi^{t}(\eta) \\
& \quad(s=j-1, j, j+1 ; t=k-1, k, k+1) .
\end{aligned}
$$

The integration span for the surface moving element is also $[-1,+1]$ in either of the directions, $\xi$ or $\eta$, around the collocation node $(j, k)$, corresponding to the territory $a_{j, k}=$ $l_{j} l_{k}$ as shown in Fig. 6. In the 3D case, if the location of the source node is near a corner or an edge of the boundary, 16-point or 12-point moving elements can be constructed, respectively, in a way similar to that in the corresponding $2 \mathrm{D}$ case.

With the introduction of the moving elements, if the source and the observation nodes are not coincident $(m \neq i)$

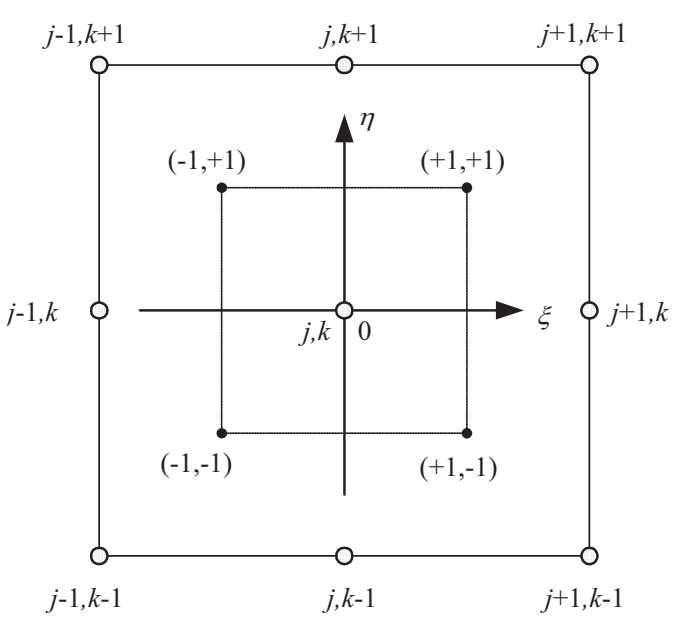

Fig. 6. The local coordinates of a 3D 'moving element'. but $r$ is relatively small, all the integrals of kernel functions in Eq. (15) can be written in the following form for both 2D and $3 \mathrm{D}$ cases:

$I_{i m}= \begin{cases}\int_{-1}^{+1} K\left[x(\xi), y_{i}\right] J(\xi) \mathrm{d} \xi & \text { (2D), } \\ \int_{-1}^{+1} \int_{-1}^{+1} K\left[x(\xi, \eta), y_{i}\right] J(\xi, \eta) \mathrm{d} \xi \mathrm{d} \eta & \text { (3D) }\end{cases}$

which can be evaluated by Gauss quadrature, where $J$ is the Jacobian, $K$ a kernel. From the process of the construction of the moving element it is evident that the shape functions under the integral sign are added together and are equal to unity because in the BPM the boundary variables are assumed to be constant within the territory of a node.

In addition to being able to describe curved boundary, with the moving element, Gauss quadrature can be carried out over the integration span if necessary. As the integration span corresponds to the territory of the collocation node only, the algorithm can be easily changed from Gauss quadrature to one-point scheme, and vice versa.

\subsection{Treatment of singular and near singular kernels}

If the source and the observation points are coincident $(m=i)$, the integral of kernel functions becomes singular. The treatment of singular kernel functions with various orders, including weakly, strongly and hypersingular, has been well documented in the BEM literature [1,22,23]. However, with the definition of the moving element and the unit property of shape function within the integration span in the BPM, the treatment of singular kernel functions becomes fairly simple. The integrals with strongly singular kernels in Eq. (15c) and hypersingular kernels in Eq. (15d) can both be evaluated indirectly with the divergence-free properties as follows:

$T_{n n}+\gamma=-\sum_{m=1, m \neq n}^{N} T_{n m}$,

$H_{n n}=-\sum_{m=1, m \neq n}^{N} H_{n m}$

The integrals in Eq. (15b) with strongly singular kernels but without the divergence-free property can be evaluated directly with the Gauss quadrature in a symmetrical manner [24]. In the 2D case, the integrals are written in the following form:

$$
\begin{aligned}
F_{i i}= & \left\{\int_{-1}^{0} \frac{\partial u^{*}\left[x(\xi), y_{i}\right]}{\partial n\left(y_{i}\right)} J(\xi) \mathrm{d} \xi\right. \\
& \left.+\int_{0}^{+} \frac{\partial u^{*}\left[x(\xi), y_{i}\right]}{\partial n\left(y_{i}\right)} J(\xi) \mathrm{d} \xi\right\}
\end{aligned}
$$

That is, the integration span is divided into two parts at the singularity point $\xi=0$ before integration, which is also the symmetrical point of the span (Fig. 7a). The Gauss 
a

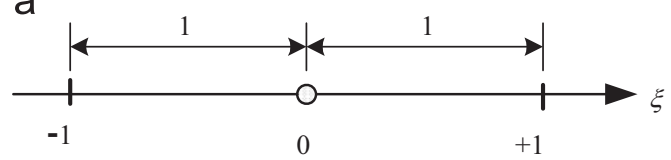

b

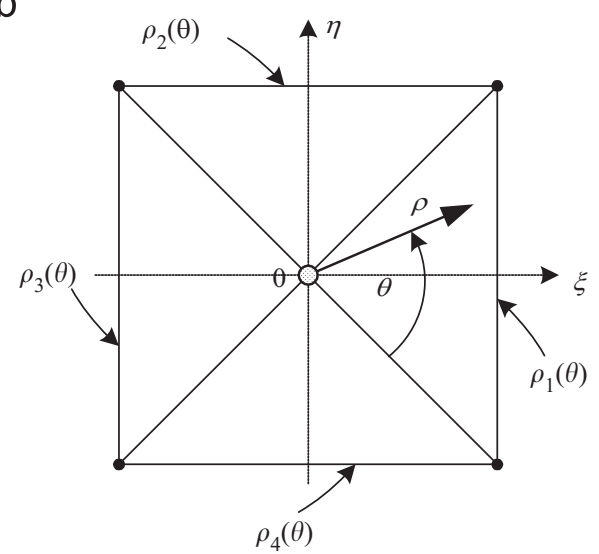

Fig. 7. Two parts in $2 \mathrm{D}$ domain (a) and four sectors in $3 \mathrm{D}$ (b) in the territory divided for symmetrical integration schemes.

quadrature is then carried out over the two parts by using the same number of Gauss points. This is because any integrand can be decomposed into two functions, an odd and an even function. Only the odd function behaves singularly and contributes to the Cauchy principal values of the integrals. However, the odd functions in the two parts will eventually be cancelled in the course of the quadrature because of the anti-symmetrical property of the odd function. In the end, only the even function contributes to the final results of these integrals.

In the $3 \mathrm{D}$ case, the integrals with strongly singular kernels without the divergence-free property can be computed with Gauss quadrature over the territory divided by four sectors in the radial-angle (Fig. 7b) coordinate system as follows [24,25]:

$$
\begin{aligned}
F_{i i}= & \sum_{l=1}^{4} \int_{\theta_{l}}^{\rho_{l}} \int_{0}^{(\theta)} \frac{\partial u^{*}\left[x(\rho, \theta), y_{i}\right]}{\partial n\left(y_{i}\right)} \\
& \times J(\rho, \theta) \rho \mathrm{d} \rho \mathrm{d} \theta \quad \text { (3D). }
\end{aligned}
$$

A similar description can be provided for decomposing the integrand into an odd and an even function in the 3D case, which is applicable with respect to the radial coordinate $\rho$. It can be seen that the proposed symmetrical integration scheme is very simple to use, with no special treatment required for evaluating integrals with the strongly singular kernels. The evaluation of singular integrals over territories of straight line in $2 \mathrm{D}$ or of flat surface in $3 \mathrm{D}$ can be further simplified, as is done in the BEM using constant boundary elements [22].

There are near singular cases when the distance between the source and the field points is very small but not coincident. The distance transformation techniques $[25,26]$ are employed in the present work to deal with the integrals in the near singular cases.

\section{Numerical examples}

To demonstrate the validity and accuracy of the proposed BPM, potential problems controlled by Eq. (1) with mixed boundary conditions (2) and (3) in elliptical and square domains in $2 \mathrm{D}$ and a quarter cylinder domain in $3 \mathrm{D}$ subject to mixed boundary conditions are considered. The field variables and their derivatives are computed at the locations in the domain, on the boundary and close to the boundary using the proposed BPM. The numerical results are compared with the exact solutions.

\subsection{Example 1: a $2 D$ elliptical domain}

The geometry and node distributions (total node number $N=60$ in this example) of the problem are shown in Fig. 8. It is subjected to mixed boundary conditions (see Fig. 8). The analytical solution of this problem is simply given as

$u\left(x_{1}, x_{2}\right)=\ln (R), \quad R=\sqrt{\left(x_{1}+a\right)^{2}+\left(x_{2}+b\right)^{2}}$,

where $a=1$ and $b=0.6$ are the major and minor half axes of the ellipse. By using the first and the second equations, respectively, with the indirect formulations of the proposed meshless BPM, the field variables $u$ and the derivatives $u_{1}=\partial u / \partial x_{1}$ and $u_{2}=\partial u / \partial x_{2}$ in the domain are computed along a straight line $x_{2}=0$. The results are plotted in Fig. 9 and compared with the analytical solution (36). It can be seen from Fig. 9 that the results from the proposed model are in good agreement with the analytical ones. With the direct formulations, the field variables and derivatives are computed along the elliptical boundary $\left(x_{1}=a \cos \theta\right.$, $x_{2}=b \sin \theta$ ). The results are listed in Fig. 10 and are compared with the analytical ones. It can be seen from Fig. 10 the results from the proposed model are again in good agreement with the analytical ones. In order to examine the effect of the near singularity, the field variables and derivatives are computed along an elliptical curve $\left(x_{1}=0.95 a \cos \theta, x_{2}=0.95 b \sin \theta\right)$ which is very close to the boundary. The results are listed in Fig. 11, showing the consistence of the computed results with the exact solutions.

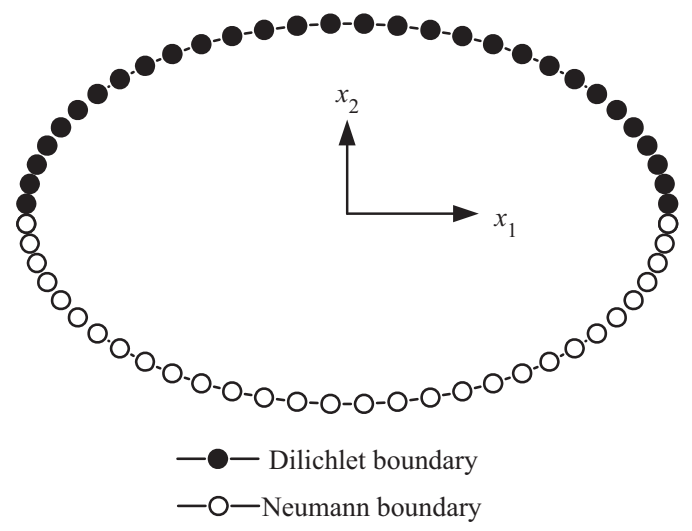

Fig. 8. The elliptical domain and boundary conditions in example 1 . 


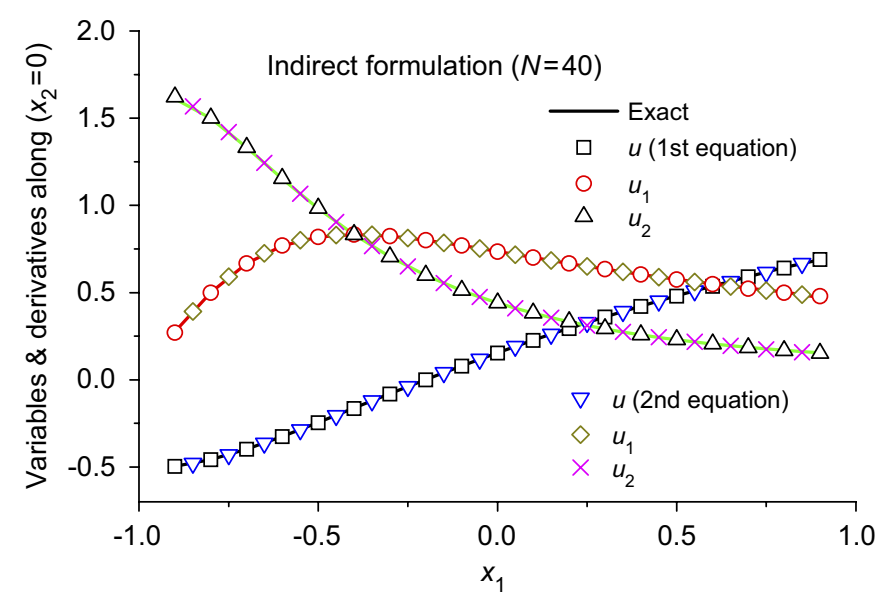

Fig. 9. Computed results in elliptical domain by indirect formulations.

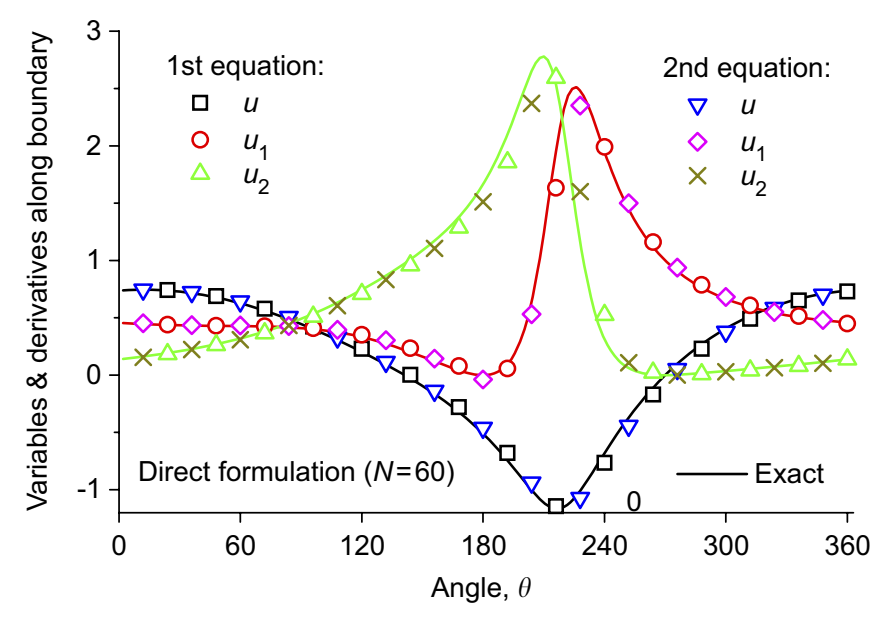

Fig. 10. Computed results on the boundary of elliptical domain by direct formulations.

To study the convergent performance of the proposed algorithm, the root mean square (RMS) error is defined as

$\mathrm{RMS}=\sqrt{\frac{1}{m} \sum_{k=1}^{m}\left(c_{k}-e_{k}\right)}$,

where $c_{k}, e_{k}$ and $m$ represent, respectively, the results from the proposed algorithm, analytical results and the total nodal number. The RMS errors as functions of the total node numbers using the indirect and direct formulations are plotted in Figs. 12 and 13, respectively, showing the convergence behaviour of the proposed BPM. The RMS error of the BPM with the second equations of either direct or indirect formulation shows stagnant at the order of about $10^{-3}$, but the corresponding results with the first equations is not stagnant, as seen in the results of Figs. 12 and 13. This phenomenon is considered to be induced from the differences between the kernels of the two equations. That is the reason why the first equation is predominantly employed in practice while the second equation is generally employed as a supplement to the first equations in some special cases such as crack problems, thin structures to

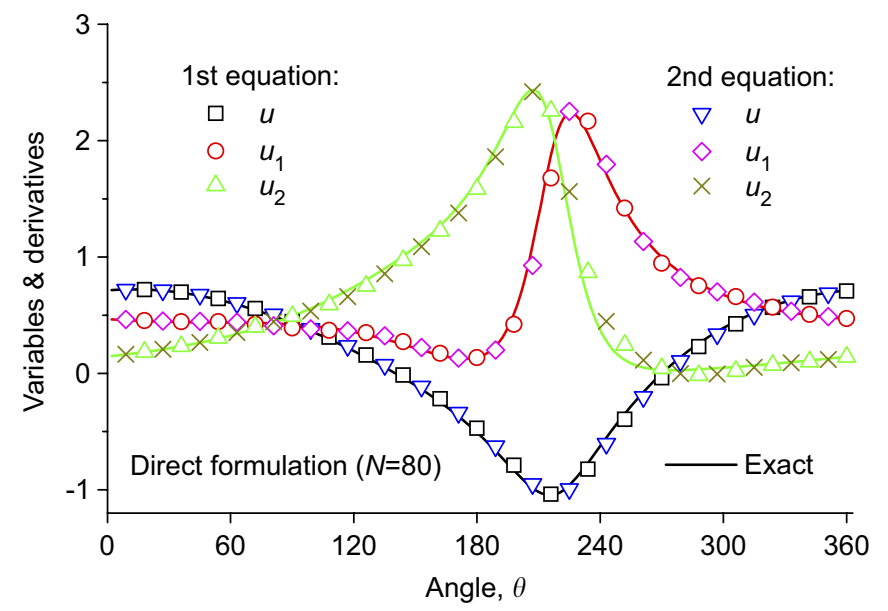

Fig. 11. Computed results at points close to the boundary of elliptical domain by direct formulations.

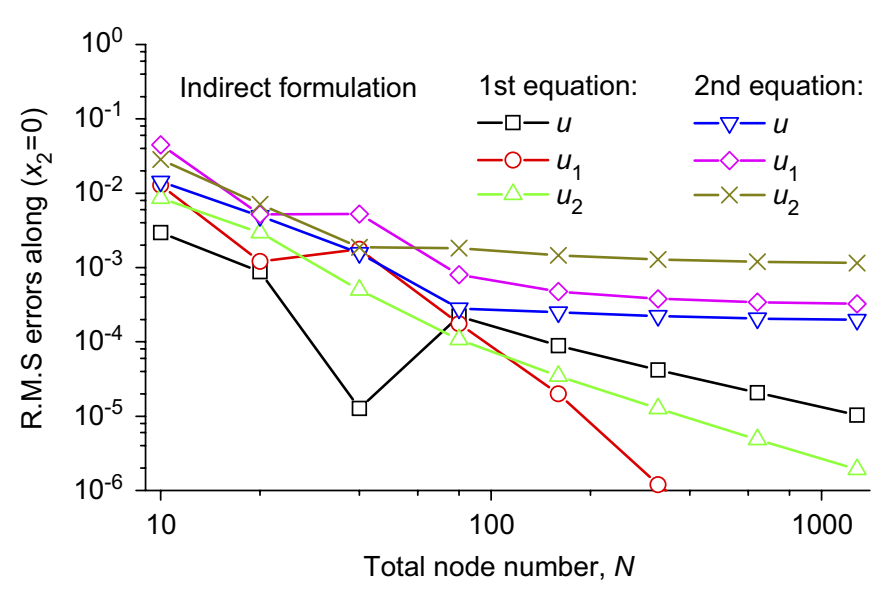

Fig. 12. Convergence of indirect formulations in terms of number of nodes.

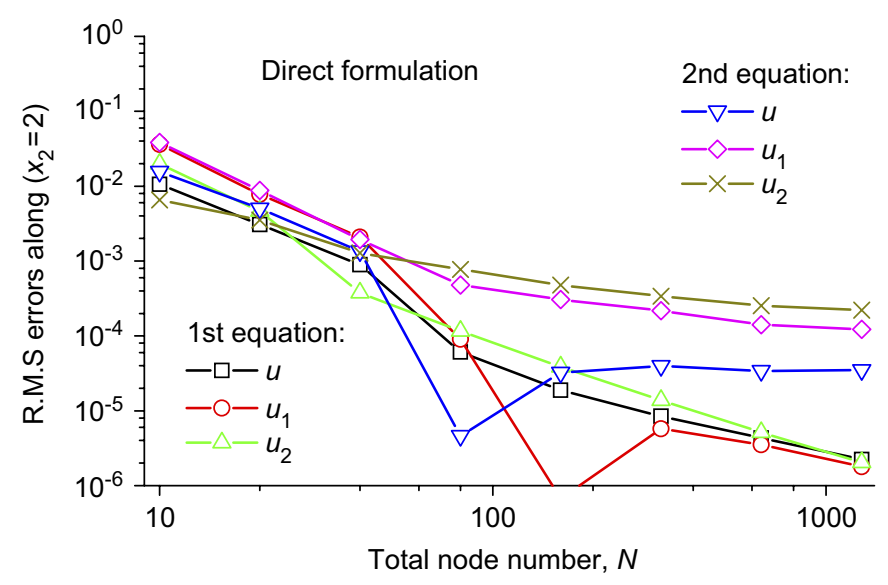

Fig. 13. Convergence of direct formulations in terms of number of nodes.

avoid the degeneration of the system matrix, as well as the direct recovery of the derivatives of field variables on the boundary. It can be seen from Figs. 12 and 13 that there are no distinct differences between the results from the first 
and the second equations, using either the indirect or direct formulations of the proposed BPM.

\subsection{Example 2: a 2D square domain}

The second example is a square domain $(1 \times 1)$ subject to the mixed boundary conditions: $u\left(1, x_{2}\right)=1, u\left(x_{1}, 1\right)=0$, $q\left(x_{1}, 0\right)=q\left(0, x_{2}\right)=0$, as shown in Fig. 14. In this case, the analytical solution of this problem is

$$
\begin{aligned}
u\left(x_{1}, x_{2}\right)= & \sum_{k=1}^{\infty} C_{k} \cosh \left[\frac{1}{2}(2 k-1) \pi x_{1}\right] \\
& \times \cos \left[\frac{1}{2}(2 k-1) \pi x_{2}\right],
\end{aligned}
$$

where

$C_{k}=\frac{4(-1)^{k+1}}{(2 k-1) \pi \cosh \left[\frac{1}{2}(2 k-1) \pi\right]}$.

In this example, only the direct formulation is employed for evaluating the first and the second equations of the proposed meshless BPM. The field variable $u$ and derivatives $u_{1}$ and $u_{2}$ in the domain are computed along a straight line $x_{1}=0.5$. The predicted results are plotted in Fig. 15 and compared with the analytical solutions. The results for the field variable and derivative along the Neumann boundary $\left(x_{2}=0\right)$ are listed in Fig. 16 and compared with the exact solutions. Further, the field variables and derivatives close to the Dilichlet boundary are evaluated along a straight line $\left(x_{1}=0.95\right)$. The results are plotted in Fig. 17, showing the consistency with the exact solutions. It can be seen again from Figs. 16 and 17 that the predicted results are in good agreement with exact solutions.

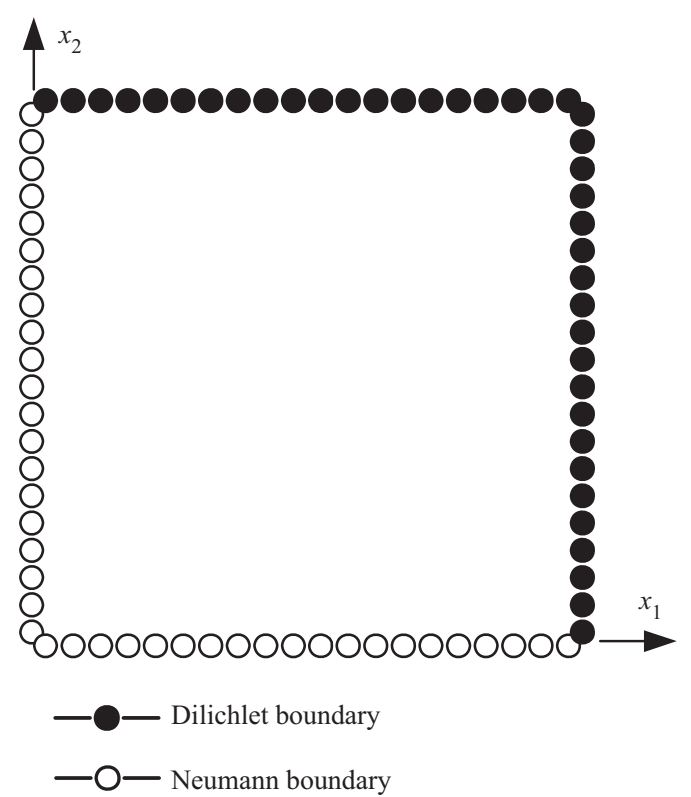

Fig. 14. The square domain and boundary conditions in example 2 .

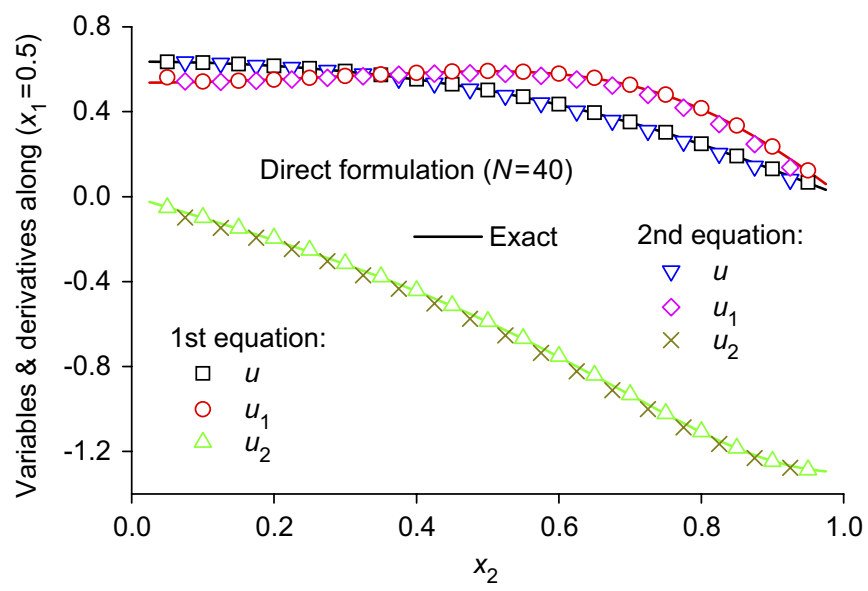

Fig. 15. Computed results in square domain by direct formulations.

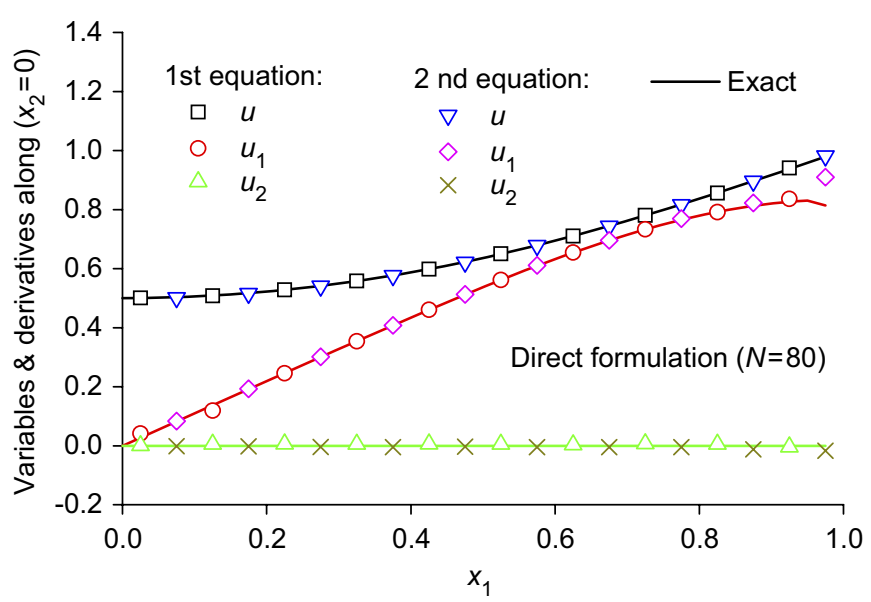

Fig. 16. Computed results on the boundary of square domain by direct formulations.

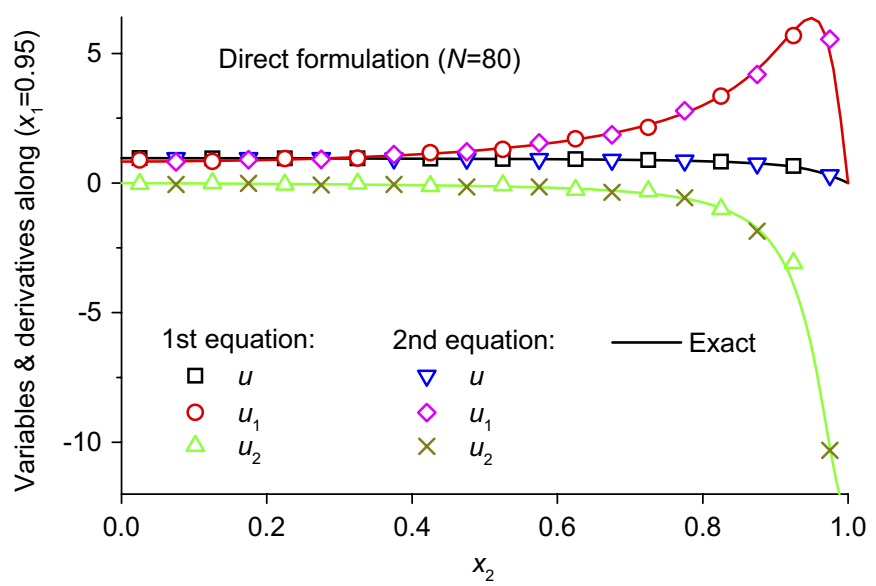

Fig. 17. Computed results close to the boundary of square domain by direct formulations.

\subsection{Example 3: a 3D quarter cylinder}

The third example is a quarter cylinder with an inner radius $a=1$, outer radius $b=2$ and thickness $h=1$ as 
shown in Fig. 18. The Dilichlet boundary condition is specified at the surface $x_{1}=0$ and $x_{2}=0$ while the Neumann boundary condition is specified at the remaining surface. The problem has an analytical solution as follows:

$u\left(x_{1}, x_{2}\right)=-\ln (R), \quad R=\sqrt{\left(x_{1}+0.5\right)^{2}+x_{2}^{2}+x_{3}^{2}}$.

In our analysis, the boundary of the cylinder is discretized by 640 boundary nodes and the problem is solved with the direct formulations only for the sake of conciseness. The field variables $u$ and $u_{k}(k=1,2,3)$ in the domain are evaluated along an arc of $x_{1}=1.5 \cos \theta, x_{2}=1.5 \sin \theta$, $x_{3}=0.5$. The predicted results in the domain are listed in Fig. 19 and compared with the exact solution (40). The field variables on the outer cylindrical surface along an arc of $x_{1}=b \cos \theta, x_{2}=b \sin \theta, x_{3}=0.56$ are also evaluated to study further the performance of the proposed algorithm. The predicted results are shown in Fig. 20 and are compared with the exact solutions. Finally, to study boundary effect, the field variables near the cylinder boundary $\left(x_{3}=h\right.$ along an arc of $x_{1}=1.4 \cos \theta$, $\left.x_{2}=1.4 \sin \theta, x_{3}=0.95 h\right)$ are evaluated and the results are listed in Fig. 21. It can be seen again from Figs. 19-21 that the predicted results are consistent with that from the exact solution (40).

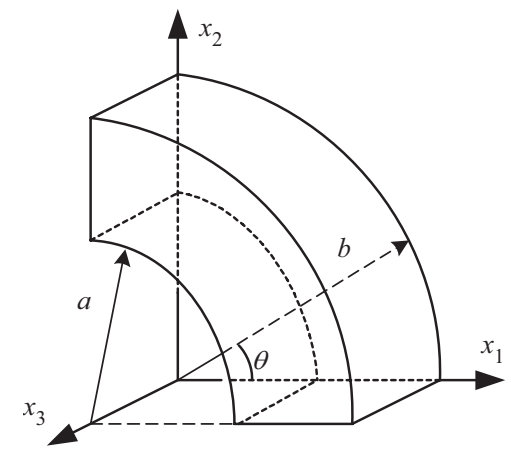

Fig. 18. The domain of a quarter of cylinder for the 3D potential problem.

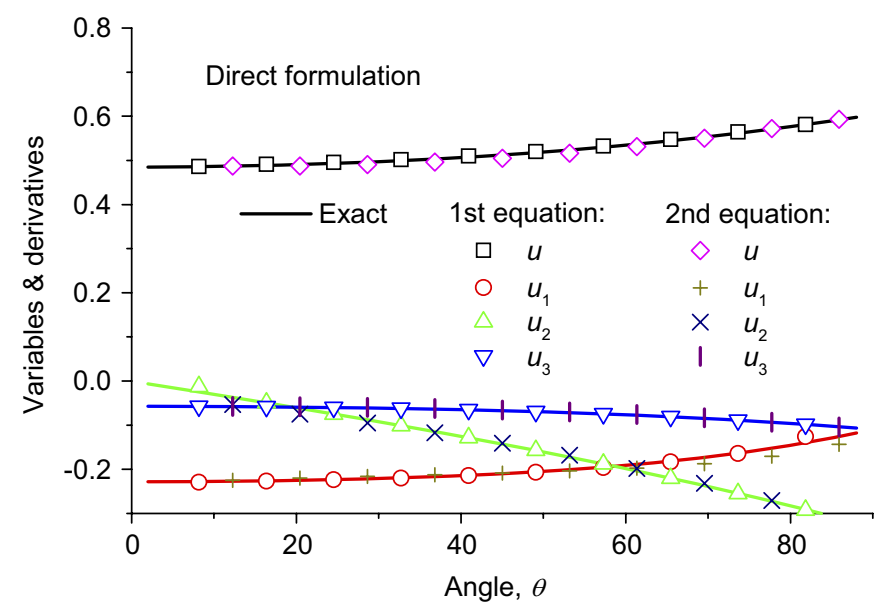

Fig. 19. Computed results in the domain of the cylinder.

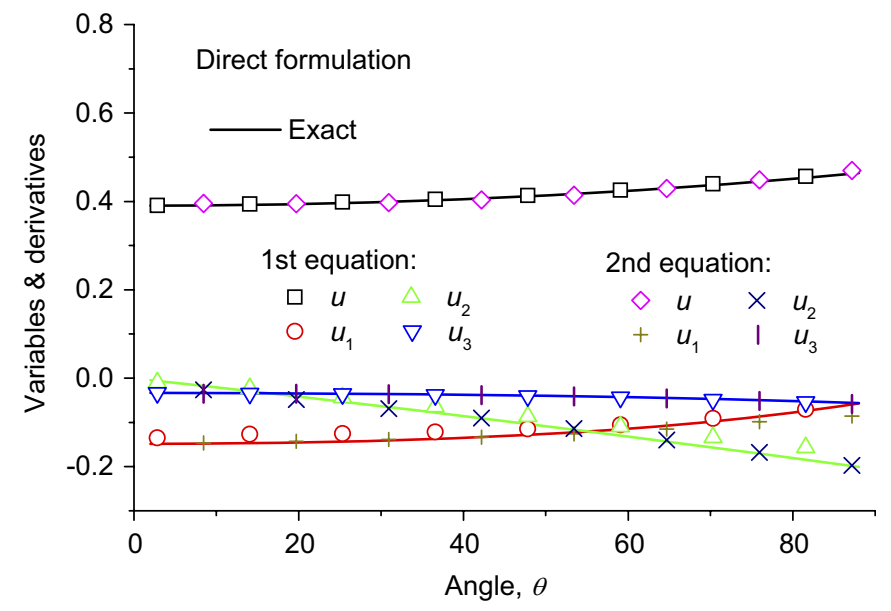

Fig. 20. Computed results on the outer surface of the cylinder.

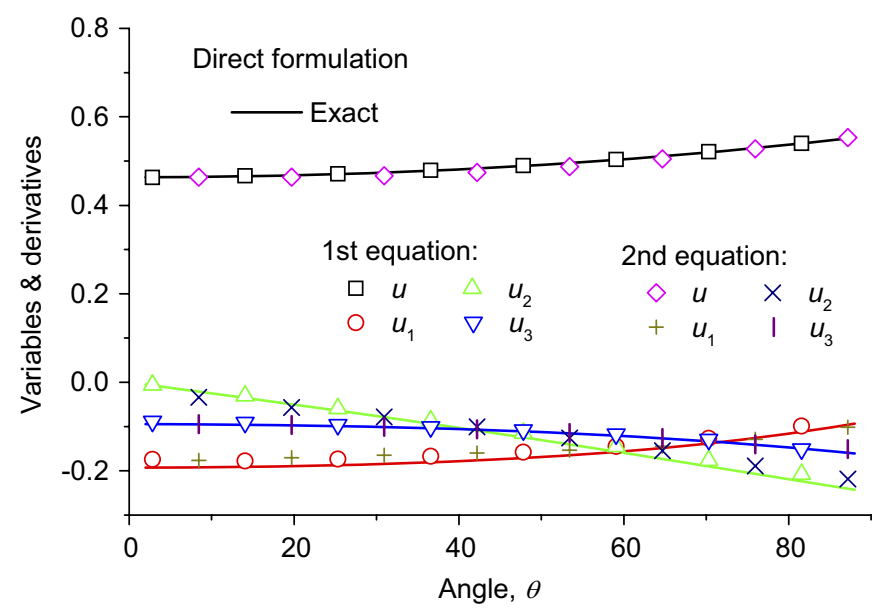

Fig. 21. Computed results at points close to the surface of the cylinder.

\section{Discussions}

As the basis of the BPM is the BIE and the idea of the BPM is stimulated by the boundary-type meshless method, especially the MFS [15-20], the BPM can be considered as a method with the features of both the BEM and the MFS, as both of them are based on the fundamental solutions. However, the BPM has features of one-point computation in which the use of fictitious boundary is not required. That is, it has the advantages of the BEM and the MFS but excludes the shortcomings of both. In [20] although both source and field points can be placed on real physical boundaries, only the kernels with the divergence-free property should be used with equally spaced node distributions along the boundary, which takes a form corresponding to the second equation of the indirect formulations in the proposed BPM. However, there is no such limitation in the proposed BPM. Further the introduction of one-point scheme to the proposed BPM will significantly improve the computational efficiency of the method. The results presented show that the use of one-point scheme can achieve 
reasonably accurate results for most of the coefficients in the system matrix. Consequently, Gauss quadrature is required only in the cases that the source and field points coincide or the distance between the two points is too small. As a result, the computational cost can be significantly saved. For example, in the 2D BPM, only the diagonal and the sub-diagonal terms of the system matrix are evaluated by Gauss quadrature, consisting $3 / N$ of the total coefficient terms only, where $N$ is the order of the system matrix. The feasibility of one-point scheme comes from the properties of the fundamental solutions that the greater the distance between the source and field points is, the smaller the values and more essentially, the smaller the variations of the fundamental solutions is. The property has not been fully explored in the literature. The discussion above is also true for other boundary-type meshless methods based on the BIE, such as the BNM $[8,9]$ where the interpolation is realized by the moving leastsquare approximation, the BPIM and BRPIM [27] where the point interpolation is realized by using polynomials or RBF as the basis functions. In these boundary-type meshless methods, however, all of the coefficient terms have to be evaluated by Gauss quadrature, which seems to be unnecessary in the proposed BPM.

There are two functions in terms of boundary nodes. The first is to describe the location and geometry of the boundary. The second is to represent the field variables and derivatives along the boundary. With the introduction of the moving elements, the geometry of the curved boundary can be described much better than that of constant elements in the BEM. In addition, the integrals of singular kernels can be evaluated using the well-established techniques in BEM and the Gauss quadrature can be carried out over the territory if necessary. As the integration span corresponds only to the territory of the collocation node in the BPM, the computational algorithm can be switched readily between the Gauss quadrature and one-point scheme. However, as the field variables over a territory are assumed to be constant, it is postulated that the accuracy of the BPM is better than that of the BEM when using the constant elements but inferior to that of the $\mathrm{BEM}$ when using the quadratic elements. It is believed that the proposed approximation derivation procedure leading to the BPM can be extended to numerous applications in science and engineering. It is also considered that the BPM would be more feasible to be combined with the fast multipole technique [28] to solve large-scale problems, which is underway.

\section{Conclusion}

In this study, a novel boundary-type meshless method, the BPM, is developed. As a numerical method, the BPM can be considered to have both the features of BEM and MFS, as it is derived via an approximation procedure based on the BIE and contains the main features of the MFS such as the one-point scheme, where no integral quadrature is required. Further, the deficiency of using a pseudo-offset-boundary in most previous MFS, which may result in ill-conditioned system matrices have been eliminated in the proposed BPM. With the introduction of the moving elements, the resulting weak singular, singular and hypersingular kernel functions can be readily evaluated by well-developed techniques in the BEM. The first and the second equations in the indirect and direct formulations are presented and tested by $2 \mathrm{D}$ and $3 \mathrm{D}$ potential problems with mixed boundary conditions.

\section{References}

[1] Brebbia CA, Telles JCF, Wrobel LC. Boundary element techniquestheory and applications in engineering. Berlin: Springer; 1984.

[2] Belytschko T, Lu YY, Gu L. Element-free Galerkin methods. Int J Numer Methods Eng 1994;37:229-56.

[3] Liu WK, Jun S, Zhang YF. Reproducing kernel particle methods. Int J Numer Methods Eng 1995;20:1081-106.

[4] Liu GR, Gu YT. A point interpolation method for two-dimensional solid. Int J Numer Methods Eng 2001;50:937-51.

[5] Wang J, Liu GR. A point interpolation meshless method based on radial basis functions. Int J Numer Methods Eng 2002;54:1623-48.

[6] Atluri SN, Zhu T. A new meshless local Petrov-Galerkin (MLPG) approach in computational mechanics. Comput Mech 1998;22:117-27.

[7] Bonet J, Kulasegaram S. A simplified approach to enhance the performance of smooth particle hydrodynamics methods. Appl Math Comput 2002;126:133-55.

[8] Mukherjee YX, Mukherjee S. The boundary node method for potential problems. Int J Numer Methods Eng 1997;40:797-815.

[9] Zhang JM, Tanaka M, Matsumoto T. Meshless analysis of potential problems in three dimensions with the hybrid boundary node method. Int J Numer Methods Eng 2004;59:1147-60.

[10] Herrera I, Gourgeon H. Boundary methods, C-complete systems for Stokes problems. Comput Methods Appl Mech Eng 1982;30:225-41.

[11] Kita E, Kamiya N. Trefftz method: an overview. Adv Eng Software 1995;24:3-12.

[12] Chen W, Tanaka M. A meshfree, integration-free and boundary-only RBF technique. Comput Math Appl 2002;43:379-91.

[13] Chen W, Hon YC. Numerical investigation on convergence of boundary knot method in the analysis of homogeneous Helmholtz, modified Helmholtz and convection-diffusion problems. Comput Methods Appl Mech Eng 2003;192:1859-75.

[14] Kita E, Kamiya N, Ikeda Y. An application of Trefftz method to the sensitivity analysis of two-dimensional potential problem. Int $\mathrm{J}$ Numer Methods Eng 1995;38:2209-24.

[15] Golberg MA. The method of fundamental solution for Poisson's equation. Eng Anal Bound Elem 1995;16:205-13.

[16] Fairweather G, Karageorghis A. The method of fundamental solution for elliptic boundary value problems. Adv Comput Math 1998;9:69-95.

[17] Wang H, Qin QH, Kang YL. A new meshless method for steady-state heat conduction problems in anisotropic and inhomogeneous media. Arch Appl Mech 2005;74:563-79.

[18] Wang H, Qin QH. A meshless method for generalized linear or nonlinear Poisson-type problems. Eng Anal Bound Elem 2006;30: 515-21.

[19] Young DL, Jane SJ, Fan CM, Murugesan K, Tsai CC. The method of fundamental solutions for 2D and 3D Stokes problems. J Comput Phys 2006;211:1-8.

[20] Young DL, Chen KH, Lee CW. Novel meshless method for solving the potential problems with arbitrary domain. J Comput Phys 2005; 209:290-321. 
[21] Gu YT, Liu GR. Hybrid boundary point interpolation methods and their coupling with the element free Galerkin method. Eng Anal Bound Elem 2003;27:905-17.

[22] Mukherjee S. Boundary element method in creep and fracture. London: Applied Science Publishers; 1982.

[23] Guiggiani M, Krishnasamy G, Rudolghi TJ, Rizzo FJ. A general algorithm for the numerical solution of hypersingular boundary integral equations. ASME J Appl Mech 1992;59:604-14.

[24] Ma H, Kamiya N. Nearly singular approximations of CPV integrals with end- and corner-singularities for the numerical solution of hypersingular boundary integral equations. Eng Anal Bound Elem 2003;27:625-37.
[25] Ma H, Kamiya N. A general algorithm for the numerical evaluation of nearly singular boundary integrals of various orders for two- and three-dimensional elasticity. Comput Mech 2002;29:277-88.

[26] Ma H, Kamiya N. Distance transformation for the numerical evaluation of near singular boundary integrals with various kernels in boundary element method. Eng Anal Bound Elem 2002;26: 329-39.

[27] Liu GR, Gu YT. Boundary meshfree methods based on the boundary point interpolation methods. Eng Anal Bound Elem 2004;28: 475-87.

[28] Greengard LF, Rokhlin V. A fast algorithm for particle simulations. J Comput Phys 1987;73:325-48. 